\section{L'Actualité économique}

L'ACTUALITÉ ÉCONOMIQUE

\section{La banqueroute de François Étienne Cugnet, 1742} III- Cugnet et les Forges de Saint-Maurice

\section{Cameron Nish}

Volume 41, numéro 4, janvier-mars 1966

URI : https://id.erudit.org/iderudit/1003146ar

DOI : https://doi.org/10.7202/1003146ar

Aller au sommaire du numéro

Éditeur(s)

HEC Montréa

ISSN

0001-771X (imprimé)

1710-3991 (numérique)

Découvrir la revue

Citer ce document

Nish, C. (1966). La banqueroute de François Étienne Cugnet, 1742 : III- Cugnet et les Forges de Saint-Maurice. L'Actualité économique, 41(4), 762-810.

https://doi.org/10.7202/1003146ar
Ce document est protégé par la loi sur le droit d'auteur. L'utilisation des services d'Érudit (y compris la reproduction) est assujettie à sa politique d'utilisation que vous pouvez consulter en ligne.

https://apropos.erudit.org/fr/usagers/politique-dutilisation/ 


\title{
La banqueroute de François Etienne Cugnet, 1742
}

\author{
III. Cugnet et les Forges de Saint-Maurice (1)
}

\section{Introduction}

Dans ce numéro de L'Actualité Économique et dans les deux suivants nous présentons des textes portant sur les Forges de SaintMaurice. Cette première tranche donne le détail de la formation de la Compagnie. La deuxième sera consacrée à la faillite de l'entre' prise et la troisième à l'administration des Forges par l'État. Après la présentation des cinq tranches de textes sur Cugnet et ses entreprises, nous tirerons les conclusions qui semblent découler de cet ensemble.

\section{Cugnet et les Forges de Saint-Maurice}

«Cession au Roy pour la securité du Sieur Francheville du privilège de l'exploitation des mines de fer du $238^{\text {bre }} 1735$. » A.P.C., Série C 11 A, Canada, Forges de Saint-Maurice, 1729-1741, vol. 110, tome 1, pp. 93-101.

Par devant le Notaire Royal en la prévoté de Quebec y résident soussigné, furent présent en leurs personnes Demoiselle Thérèse de Couagne veuve de feu François Poulin de Francheville demeurante ordinairement a Montréal rue Saint Paul, de présent en cette ville, Sieur Pierre Poulin marchand de Québec y demeurant rue sous le fort, Sieur Ignace Gamelin négociant à Montréal y demeurant ordinairement de présent en cette ville, et Sieur François Estienne $\mathrm{Cu}$ gnet Directeur du Domaine du Roy en ce pays demeurant à Quebec au Bureau du dit Domaine, rue Saint Pierre tous intéressés en l'ex' ploitation des mines de fer dans l'étendue de terrain depuis et compris la Seigneurie d'Yamaschiche jusques et compris la Seigneurie du cap de la Madeleine suivant et aux termes du privilège accordé 
au dit Sieur François Poulin de Francheville par Brevet de Sa Majesté du vingt cinq Mars gbyc $\mathbf{x x x}^{1}$ et arrest du Conseil d'Etat du quatre aoust au dit an, lequel privilège le dit feu Sieur François Francheville a cédé a la Compagnie des intéressés cy-dessus nommés par traité de Société passé entre le dit feu François Poulin de Francheville et les dits Sieurs Pierre Poulin Ignace Gamelin et François Estienne Cugnet sous leurs signatures privées le Seize Janvier mil sept cent trente trois, reconnu par acte passé devant le notaire soussigné le mế me jour, lesquels intéresses de leur bon gré et volonté reconnoissant qu'ils ne sont point en etat de continuer l'exploitation des dites mines a cause des fonds considérables qu'il est nécessaire de faire pour l'établissement des forges a l'effet de retirer des dites mines l'utilité qu'elles peuvent procurer suivant le projet qui leur en a été présenté dressé par Monsieur Olivier de Vézain maitre de forge envoié en ce pays par Monseigneur le Comte de Maurepas pour les dites mines et que les établissements faits par le feu Sieur de Francheville pour la dite société ne peuvent servir a ceux qui le dit Sieur Olivier propose : ont par ces présentes remis et remettent à $\mathrm{Sa} \mathrm{Ma-}$ jesté et mains de Messire Gilles Hocquart Chevalier Conseiller du Roy en ses conseils Intendant de justice Police et Finances en cette Colonie Le privilège accordé par Sa Majesté au dit feu Sieur Fran' çois Poulin de Francheville par le dit Brevet du vingt cinq Mars gbyc trente et arrest du Conseil d'Etat du quatre aoust au dit an pour en disposer par Sa Majesté en faveur de qui et ainsi qu'elle le jugera a propos, se désistant les dits Sieurs et Demoiselle comparant du dit privilège, et renonçans tous les droits généralement qu'ils ont et peuvent avoir et prétendre en vertu des dits Brevet et arrest du Conseil des vingt cinq Mars et quatre aoust gbyc trente comme aussi les dits Srs et Demoiselle comparant ont cédé, quitté, et remis cèdent, quittent et remettent à Sa Majesté ou à qui elle voudra accorder le dit privilège la seigneurie de St Maurice acheté par le dit Traitté de société du seize Janvier mil sept cent trente trois a constitution de trois cent livres de rente rachatable a toujours au principal de six mille livres, ensemble tous les Batimiens, la forge, les harnois des dites forges, dalles et chemins d'eau, maisons meubles outils mine charbon ustansils, et tous les effets meubles et immeubles géné

I. Le 25 mars 1730. 


\section{IACTUALITE ECONOMIQUE}

ralement quelconques qui sont actuellement au lieu de l'établissement fait sur le ruisseau de St Maurice par le dit feu Sieur Franche: ville : les dites cession et abandon fait aux conditions que Sa Majesté sera très humblement suppliée de vouloir décharger les dits Sieurs et Demoiselle interessés des obligations consenties par le dit feu Sieur Francheville devant Louet Notaire à Quebec le onze octobre mil sept cent trente deux et par la dit Demoiselle veuve Francheville devant Gaudron de Chevremont et son confrère Notaires a Montréal le 19 décembrbe mil sept cent trente trois de la somme de dix milles livres avancées des deniers de Sa Majesté au dit feu Sieur Franche' $\overline{v i l l e}$ pour lui faciliter les moiens d'exploiter les $\mathrm{d}$. mines du payement de laquelle somme de dix mille livres et autres dettes contractés pour raison desd. mines la dte Delle veuve Francheville et les d Srs Poulin Gamelin et Cugnet. Demeureront bien et valablement quittes et dé, chargés envers Sa Majesté sans pouvoir en etre inquiéter ny rechercher a l'avenir : que la d. delle veuve Francheville et les $d$. Srs Poulin Gamelin et Cugnet seront remboursés chacun suivant son intérest en la d. Société du 16 Janvier 1733 par Sa Majesté ou ceux qui obtiendront d'elle le privilège des d. mines de toutes les depenses par eux faites pour l'établissement des d. forges de St. Maurice suivant le compte qui en sera réglé et arresté avec les $\mathrm{d}$. Sieurs et delle comparants par qui et ainsy qu'il appartiendra ce qu'il sera passé par Sa Majesté ou ceux qui obtiendront d'elle le privilège des dites mines au profit du dit Sieur Pierre Poulin, des héritiers du dit feu Sieur François Poulin et Francheville et autres co propriétaire de la Seigneurie de Saint Maurice contract de constitution de trois cent livres de rente rachetable au principal de six mille livres pour l'acquisition de la dite Seigneurie de Saint Maurice, laquelle sera vendue et cédée à Sa Majesté ou a ceux qui obtiendront d'elle le privi. lège des dites mines par les co propriétaires en toute propriété, fonds trés fonds, Droits, noms, raisons et actions au moyen de la dite constitution de toir cent livres de rente rachetable au principal de six mille livres, et au moyen des dites conditions cy-dessus les dits sieurs et Demoiselle comparants promettent renoncer au privilège accordé par le dit Brevet et arrest du Conseil d'Etat des vingt cinq Mars et quatre aoust mil sept cent trente et a tous les droits qu'ils peuvent avoir et prétendu tant sur la dite Seigneurie de Saint Maurice que sur tous les établissements faits au dit lieu de Saint Maurice 
pour l'exploitation des dits mines, maisons, meubles, outils, ustancils, mine et charbon, et autres effets généralement quelconques y étand. Car ainsi \&a promettant $\mathcal{\mho}^{2} a$ obligeant $\varepsilon^{2} a$ Renonçant \&a

Fait et passé en l'hotel de Monseigneur l'Intendant le vingt trois octobre mil sept cent trente cinq avant midy en présence des Sieurs François Emmanuel Moreau Derosier et Florent Derosier témoins demeurant au dit Québec qui ont avec les dits Demoiselle veuve Francheville Poulin Gamelin Cugnet mon dit Seigneur l'Intendant et Notaires signé lecture faite Suivant lordonnance ainsy signé De Couagne Vve Francheville. P. Poulin I Gamelin, Cugnet, Hocquart, Moreau, F. Derosier et Pinguet notaire avec paraphe. 1 t a la minutte/

Pinguet

«Offres et sousmissions par les Sieurs Cugnet, Olivier de Vezain et Gamelin de se charger de l'Etablissement des forges de Saint Maurice... » A.P.C., Série C 11 A, Canada, Forges de Saint-Maurice, 1729-1741, vol. 110, tome 1, p. 102-108.

Offres et sousmission par les Sieurs Cugnet, Olivier de Vezain et Gamelin de se charger de l'Etablissement des forges de Saint Maurice et de l'exploitation des mines de fer en leur accordant un privilège de 20 années.

A condition que le Roi leur fera une avance de cent mille livres pour les construction des Batimens necessaires à l'exploitation

S'obligeant les sousmissionnaires au remboursement de cette somme et celle de $10000^{\prime \prime}$ prêtée au Sieur Francheville aiant cy devant le privilège

Nous soussignés Pierre François Olivier de Vezain maitre de forge, Ignace Gamelin négociant à Montreal et François Estienne Cugnet Directeur du Domaine d'Occident en ce pays, en conséquence de l'acte passé aujourd'hui devant Pinguet notaire à Quebec par lequel la Demoiselle veuve Francheville, le sieur Pierre Poulin, et les Sieurs Gamelin et Cugnet cy devant intéressés en l'exploitation des mines de fer dont le privilège avoit été accordé par Sa Majesté au feu Sieur François Poulin Francheville par Brevet du 25 mars et arrest du Conseil d'Etat du 4 aoust 1730 ont abandonné et remis à Sa Majesté es mains de Monsieur l'Intendant le dit privilège ensemble la Seigneurie de Saint Maurice avec les Batimens 
forge, maison, meubles, outils ustancils, mine charbon et autres effets meubles et immeubles généralement quelconques étant sur la dite seigneurie de Saint Maurice, et etablissement fait par le dit feu Sieur Francheville pour l'exploitation du dit privilège pour les raisons et aux conditions enoncées au dit acte, offrons à Sa Majesté et nous soumettons de nous charger de l'établissement des forges pour l'exploitation des dites mines de fer suivant le projet qui en a eté dressé par Monsieur l'Intendant le dit Sieur Olivier de Vezain et nous Gamelin et Cugnet et de continuer d'exploiter et faire valoir les dites mines, s'il plaist à Sa Majesté en revoquant le privilège accordé au dit feu Sieur Francheville par les dits brevet du 25 Mars et arrest du Conseil du 4 Aoust 1730 Nous accorder un nouveau privilège des dites mines aux mêmes termes, clauses et conditions du dit Brevet et arrest du Conseil pendant le tems et espace de vingt années consécutives a compter du jour que le fourneau aura eté chargé pour la premiere fois dont sera dressé procès verbal par le Sieur Lieutenant general de la ville des Trois Rivières, et nous prester en monnoye de cartes les fonds nécessaires suivant le dit pro jet tant pour l'etablissement et construction du fourneau de fonte, forges, affinerie chaufferie harnois et Batimens nécessaires pour l'ex' ploitation des dites mines que pour les dépenses annuelles de la dite exploitation, lesquels fonds jusqu'a concurrence de la somme de cent mille livres, Nous seront remis au fur et a mesure que nous en auront besoin, sçavoir dans la premiere année pour la construction des fourneaux, forges, harnois et Batimens, dans la seconde année pour la fabrication et transport de charbon, amas et transport de mine, castine et autres matieres et dans la troisième pour les gages des ouvriers.

Au moyen de quoy nous promettons et nous obligeons de rembourser à Sa Majesté tant les dix mille livres prester au feu Sieur Francheville suivant les obligations des onze Octobre 1732 et 19 Décembre 1733. et autres sommes qui pourroient avoir été avancées des Deniers de Sa Majesté et d'en faire le remboursement en fer provenant des dites forges ou en monnoye de cartes ainsi qu'il plaira à Sa Majesté, et au cas qu'elle accepte le remboursement en fers, ils seront par nous fournis à Quebec a raison de dix livres par millier au dessous des prix qu'ils sont acheter en france pour la marine, de tel échantillon qu'ils nous seront demander à l'exception 
neanmoins des fers de fonderie qui ne pourront nous etre demander qu'apres que nous auront établi une fonderie, a commencer le premier remboursement dans la premiere année que les forges auront travaillé et produit du fer, et continuer d'année en année jusqu'a parfait remboursement pour la sureté desquels remboursement, Nous promettons consentir obligation au profit de Sa Majesté de la dite somme de dix mille livres pretée au feu Sieur Francheville et des autres sommes que nous recevrons au fur et a mesure qu'elles nous seront remises payables aux termes cy dessus sous l'hypotèque spéciale des Batimens et fonds des forges et des fers qui en proviendront et généralement de tous nos biens meubles et immeubles présent et avenir.

Nous nous obligeons pareillement de rembourser à la Société du Sieur Francheville les dépenses par elle faites jusqu'a présent à l'oc' casion des dites mines, et de passer contract de constitution de trois cent livres de rente rachetable au principale de six milles livres pour l'acquisition de la Seigneurie de Saint Maurice aux termes du dit acte de ce jour.

Fait à Quebec le vingt trois Octobre mil sept cent trente cinq./

$$
\begin{gathered}
\text { Olivier de Vezain Cugnet } \\
\text { Ignace Gamelin }
\end{gathered}
$$

«Projet des dépenses a faire pour l'établissement et exploitation des forges de fer en Canada. », A.P.C., Série C 11 A, Canada, Forges de Saint Maurice, vol. 110, tome 1, pp. 323-334.

\section{Premièrement}

Depenses a faire pour l'Etablissement Construction de fourneau.

Pour la masse du fourneau de 26 pieds de largeur sur 26 pieds d'épaisseur et 28 pieds de hauteur les fondations comprises le tout tant plein que vuide faisant 87 toises cubes $1 / 3$ à 50 \# la toise cube la somme de

4366.13.4.

Pour les grandes courtines de 20 pieds de largeur sur quatre faces sur 2 pieds 
d'épaisseur 11 pieds de hauteur faisant 24 toises courantes $4 / 9$ a 16 \# la toise cou rante

Pour les petites courtines de 10 pieds de largeur sur quatre faces sur 1 pieds.$/ 2$ d'épaisseur et 11 pieds de hauteur faisant Toise courante 12 toises $2 / 9$ a $16 \#$ la toise courante

195.11 .1$.

10.000 de briques recuite pour les ap paroys a 40 \#. le mille

Pour les pierres a feu pour les faces du fourneau

Les conduits pour dessecher la face du fourneau

Total de la maçonnerie

Le hallage du fourneau sur la face de la Tuyère pour mettre les soufflets et les Toues a couvert le dit hallage de pièces sur pièces de 35 piẹds de profondeur et 20 pieds de hauteur couvert en planches et en bardeau cy

Le hangard du fourneau sur la face de la coulée de pièces sur' pièces de 30 pieds de large sur 30 pieds de profondeur et 20 . pieds de hauteur couvert en planches et en bardeau cy

Les souffles du fourneau,

La Tuyère, Beures et bassecoudes

Le harnois du fourneau consistant en. ses roues rouets et lanternes

Pour les Ringards et autres outils

Pour le bief, chaussée décharges, dalles et chemins d'eau

Halle au charbon de fourneau de 60 pieds de longueur sur 40 pieds de largeur et 10 pieds de hauteur sous la charpente

$\frac{600 " \prime}{800 " \prime \prime}$
$\frac{300 " \prime \prime}{6}$
$\frac{600 " \prime}{400 " \prime}$
$\frac{1500 " \prime}{\prime \prime}$


couvert en planches et bardeau entouré de pieds debout

$$
\frac{1200 " \prime \prime}{12253.6 .8 \cdots 12253.6 .8 .}
$$

Pour un boccard et lavoir pour mine avec la roue et les mouvemens

Forge composée dé deux affinerie, une chaufferie, une taulerie, avec tous les harnois et ordous du marteau et du martinet

Le hallage de la forge de 90 pieds de longueur sur 40 pieds de largeur et quinze pieds de hauteur sous la charpente de pièces sur pièces couvert en planche et en bardeau'

Pour le solage en maçonnerie de 10 pieds de hauteur les fondations comprises sur 3 pieds d'épaisseur faisant toise cou rante $108^{\tau} \mathrm{x} / 3$ a 12 \#. la toise

Pour la charpente et couverture

Pour les appentis aux deux costés du hallage pour loger les roues des moulins de 90 pieds de longueur sur. 10 pieds de lar. geur et 10 pieds de hauteur de pieux debout couvert en planches et bardeau

$$
\frac{1300 \cdots " \prime \prime \prime \prime}{2000 \# " \prime \prime \prime}
$$

Quatre feux sçavoir deux d'affinerie, un de chaufferie et un de Taulerie chacun de 8 pieds de maçonnerie en carré sur 1 pied $1 / 2$ d'épaisseur et le pieds de hauteur les fondations comprises, leurs cheminées de 30 pieds de hauteurs sur un pied d'épaisseur par le bas en diminuant d'un demi pied jusqu'a sa hauteur qui ne trouvera que d'un pied et demi en carré par le haut.

Pour la maçonnerie de chaque feu a 10 toises $2 / 3$ chacun faisant en tout toise cour rante 42 toises $2 / 3$ a 12 \#. la Toise 512 \#

Pour les cheminées a raison de 6 Toises $2 / 3$ chacun faisant en tout Toise courante $25^{\tau} 2 / 3$ à 12 \# la Toise

Pour les quatre paires de soufflets et: garnitures

Pour les Tuyères, beures, et bassecoudes

$$
820 \#
$$

1000 \#" "

400 \# 


\section{LACTUALITÉ ECONOMIQUE}

Pour les Tacques de fonte servant aux ouvrages

Pour les ringards Tenailles et autres outils

Pour les quatre harnois des dits feux consistantes leurs arbres et roues

Pour l'ordon du marteau

Pour le marteau de fer fondụ

Pour l'enclume de fonte

Pour les liens et Touvillons de l'arbre du marteau

Pour l'ordon du martinet

Pour le marteau de fer battu

Pour l'enclume de fonte

Pour le bief avec sa chaussée décharge dalles et chemins d'eau

$$
\begin{aligned}
& 100 \# " \prime \prime \\
& 600 \# " \prime \\
& \hline
\end{aligned}
$$

La halle au charbon de 60 pieds de longueur sur 40 pieds de largeur sous la charpente entourée de pieux debout couverte en planche et bardeau

'Une boutique de maréchal de 15 pieds en carré sur 8 pieds de hauteur de pièces sur pièces couvertes en planches et barde. reau'

Pour le soufflet

Pour l'enclume

Pour la forge et cheminée $100 \# "$ "."

\begin{tabular}{|c|}
\hline $500 \# " 1$ \\
\hline $200 \#^{\prime \prime \prime}$ \\
\hline
\end{tabular}

$\frac{1200 \# " "}{1000 \# " " ~}$
$\frac{500 \# " "}{100 \# " 1 "}$

$$
\underline{2000 \# " ! "}
$$

Pour les outils

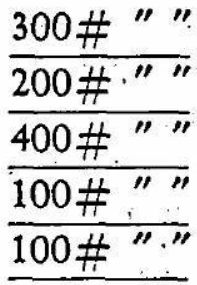

Pour les moules a marmites et autres ouvrages de fonte

Fenderie pour faire du fer de tout échantillon ${ }^{2}$

2. L'article cy contre n'est porté hors ligne parce que la fenderie ne s'etablira que lors quon aura la débouché certaine des fers en forges en feuillards et autres ouvrages de fonderie de tout échantillon. (Ce texte apparaît dans la marge de gauche sur le manuscrit). 
Le hallage de 100 pied de longueur sur 36 pieds de largeurs et 15 pieds de hauteur de pièces sur pièces couvert en planche et bardeau cy

Deux appentis pour mettre les roues a couvert de 20 pieds de longueur sur 10 pieds de largeur et 15 pieds de hauteur entourés de pieux debout couverts en plan. che et bardeau

Pour le solage sur lequel portent les roues 32 toises $2 / 9$ toise courante de maçonnerie a 12 \# la toise

Pour les roues rouets et lanternes arbres de roues liens et tourillons.

Pour les taillans moufles et appentis avec tous les ferremens et outils nécessaires

Pour le bief dechargeoir dalles et che min d'eau

$2400 \# " 1 "$

\begin{tabular}{l}
$150 \#$ \\
$386 \# 13.4$ \\
\hline
\end{tabular}

900\#.

Four a chauffer le fer defente au feu de reverbere de 15 pieds de longueur sur douze pieds de largeur 12 pieds de hauteur les fondations comprises et un pied $1 / 2$ d'épaisseur et sa voute 20 toises courantes a 12 \# la toise 240\#." "

Pour le grillage et couvertures en fonte $100 \# " 1 "$ $1000 \#$

Une boutique de maréchal pareille a celle de la forge cy

$\begin{array}{ll}\text { Pour un etoc } & 100 \# " 1 " \\ \text { Pour limes et autres outils } & 100 \# " 1 "\end{array}$ $340 \# " \prime "$

Pour la maison du fondeur et de ses: quatres ouvriers

Seize chevaus de harnois pour les travaux et voitures a faire a.150\# pièce $\frac{1000 \#^{\prime \prime \prime}}{7976 \# 13.4}$ 1300 \#" " 
Une maison pour le maitre des forges de 40 pieds en carré sur 20 pieds de hauteur a deux etages de pièces sur pièces lattée et renduite dedans et dehors couverte en planche et bardeau

Un magasin a loger les fers de 30 pieds en carré sur 8 pieds de hauteur de pièces sur pièces couvert en planches et bardeau

Une écurie

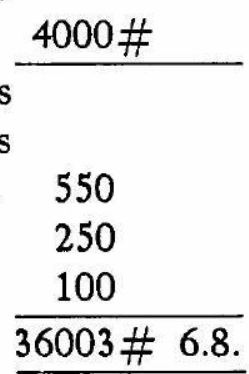

Un four

L'on ne comprend point dans ce projet la maison pour loger les forgerons et ouvriers parce que celle qui est deja construite a Saint Maurice servira, les autres bastimens ne peuvent subsister attendu qu'ils occupent la place des bastimens a faire suivant ce projet, Les materiaux pourront servir. La valeur n'en est point portée sur le présent Etat. Elle remplacera les dépenses extraordinaires qu'on n'a pû prévoir dans ce projet, tels que les chemins fouilles de terre et autres ouvrages./

Non signé

(Olivier de Vézain?)

«Dépenses annuelles de l'exploitation. », A.P.C., Série C 11 A, Canada, Forges de Saint Maurice, vol. 110, tome 1, pp. 335-339.

Le fourneau pourroit travailler pendant toute l'année et produiroit 1600 milliers de fonte dont on ne pourroit employer dans deux affeneries que 900 milliers pour faire $600^{\mathrm{m}}$ de fer en barre Ainsi il resteroit $700^{\mathrm{m}}$ de fonte, et comme l'on n'en sçait point encor la débouche on reduit le projet a huit mois de travail pour avoir $1000^{\mathrm{mrs}}$ de fonte dont on emploiera $900^{\mathrm{mrs}}$ aux affineries et il en restera $100^{\mathrm{mrs}}$ qui seront emploiés en ouvrages de fonte pour la consommation de la Colonie.

Pour $1000^{\mathrm{mrs}}$ de fonte il faut.

2000 pipes de mine a $3 \#$ la

pipe rendue au fourneau cy $6000 \#$

20.000 pipes de charbon ren-

du au fourneau a $20^{\text {s }}$ la pipe 20000 . 
1000 pipes de castine a $20^{\mathrm{s}}$ la

pipe

1000.

600 pipes de boussage a her.

bue a $20^{\mathrm{s}}$

600.

100 de suif a $10^{\text {s }}$

$$
\frac{50 .}{27650 \#}
$$

\section{Gages}

Un maitre

de forges

Un commis

$$
\begin{array}{lrr}
\text { de forges } & 3000 \# \\
\text { Un commis } & 700 & \\
\text { Un fondeur } & 1500 & \\
\text { Un petit fondeur } & 400 & \\
\text { Quatre chargeurs } & & 6800 \# \\
\text { a 300\# chacun } & 1200 & \\
\cline { 2 - 3 } & \multicolumn{3}{|c}{34.450 \#}
\end{array}
$$

Un petit fondeur 400

Quatre chargeurs

a 300 \# chacun

Les 1000 milliers de fonte reviendront a pareille somme a raison de $34 \# 9^{3}$ le mille. Cette dépense n'est cy portée hors ligne attendu qu'elle sera cy après portée aux dépenses des affineries et des ouvrages de fonte.

Depenses des affineries et chaufferies

900 milliers de fonte a 34\# le mille

$$
\begin{array}{r}
31.005 \# \\
14400 \\
\hline 45405 \#
\end{array}
$$

14400 pipes de charbon a $20^{\text {s la pipe }}$

\section{Gages}

Un marteleur $^{8} \quad 1200$ \#

Trois chauffeurs

a 600 \# chacun 1800

Un valet de chaufferie

Un affineur

1200

Sept valets

d'affinerie a 600 \# chacun

3. Ces ouvriers travailleront a la taulerie lorsqu'il en sera besoin. (Ce texte apparait dans la marge de gauche sur le manuscrit). 
Un charpentier . $\quad 500$

Un forgeron marechal

$55105 \#$

Le fer en barre reviendra sur ce pied à $91 \# 16^{\mathrm{s}} 10^{\mathrm{d}}$ le mille.

Depenses des ouvrages de fonte.

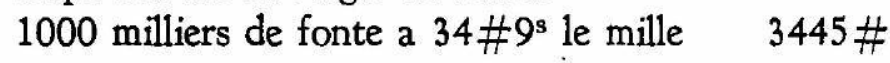

\section{Gages}

Un mouleur

$1500 \#$

Deux ouvriers a 600 \# chacun

$\begin{array}{lll}1200 & 2700 \# & 6145 \\ \ddots & 6145 \# & \\ & & \end{array}$

Dépense de la fonderie 4

Un fendeur

Quatre ouvriers a 300 \# chacun

\section{Gages}

Produit des dites forges.

Produit des affineries et chaufferies.

600 milliers de fer en barre dont il se consommera dans la colonie :

$200^{\mathrm{mrs}}$ a 200 \# le mille 40.000 .

$400^{\mathrm{mrs}}$ seront envoiés en france a

$$
\begin{aligned}
& 1500 \# \\
& \frac{1200 \#}{2700 \#}
\end{aligned}
$$

$600^{\text {mrs }} 140$ \# le mille

56000

96000

Produit des ouvrages de fonte.

$100^{\mathrm{mrs}}$ en poesles, plaques, marmites, et autres ou vrages de fonte qui se consommeront dans la Colonie

a $200 \#$ le mille

Non signé

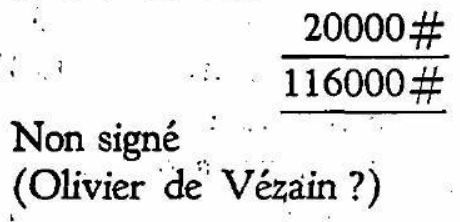

4. Cette dépense n'est portée hors ligne attendu que la fonderie sera seulement établie lorsqu'on aura la débouche certaine des fers de fenderie de tout echantillon ( $\mathrm{Ce}$ texte apparaît dans la marge de gauche sur le manuscrit). 
«Projet des sommes qu'il faut pour faire et parfaire sept cent milliers de fer et batir la petite forgette en question, suivant le détail cy après. », A.P.C., Série C 11 A, Canada, Forges de Saint Maurice, vol. 110 , tome 1 , pp. $340-344$.

\section{Primo.}

Il faut couper dix mille cordes de bois qui couteront vingt sols la corde total dix mille livres cy

Pour dressage des gens emploiés tant pour voiturer le bois en place que pour dresser et faire les places a four: neaux quatre mille cinq cens livres cy

Pour cuisinage des dix mille cordes qui produiront deux mille trois cens bannes de charbon a quarante sols la banne, quatre mille six cens livres cy

Pour feuillages des dix mille cordes quatorze cent quarante livres cy

Pour charoia des dites deux mille trois cens bannes de charbon a trente sols la banne trois mille quatre cent cinquante livres cy

Pour faire sept cens milliers de fer il faut un million cinquante milliers de fonte pour gages du fondeur a vingt cinq sols le millier treize cent soixante deux livres dix sols cy

Pour les gages de quatre hommes pour charger le fourneau a quarante livres par mois il faudra huit mois pour faire les dites fontes, six cent quarante livres cy

640.

Pour les relaveurs de mines et celui qui casse la cas. tille et celui qui la charie idem

Pour le garde fourneaux cinq cens livres cy

Il faut deux mille pipes de mines pour parfaire les fontes cy dessus mentionnées six mille livres cy

$1362 \# 10$

Pour la façon de sept cent milliers de fer a douze livres le millier huit mille quatre cent livres pour le salaire des forgerons a gages compris

8400 .

Pour la dépense de la forge douze mille livres pour le rendre faitte et parfaitte

Total se monte a la somme $12000 \#$ de cinquante trois mille cinq cent trente deux livres 
pour toute la dépense susdite les sept cens milliers de fer qui proviendront de cette dépense, lesquelles seront ven. dus au pays a deux cens livres le millier produiront cent. quarante mille livres au moins. Il y aura de profit sur deux années que l'on employera pour fabriquer ces fers. quatre vingt six mille quatre cent soixante huit livres cy 86468

C'est un objet asses considérable pour y faire attention et d'ailleurs qui bonifira pour toujours notre entreprise. sans lequel secours tout perira, si l'on ne suit ce projet. Il sera très aisé de le faire réussir par un simple arrangement que $\mathrm{M}^{\mathrm{r}}$ l'Intendant nous permettra de prendre avec luy. qu'il trouvera de son gout au mo ment qu'il daignera écouter nos justes raisons comm'il n'y a aucun fonds pour parvenir a faire cette entreprise et les apprèts en conformité du présent projet. Il faut livrer deux cent milliers de fer a Monsieur l'Intendant qui nous procurera ces fonds a fur et mesure que l'on en aura besoin pour travailler en lui foumissant exactement et fidèlement des mémoires de l'ouvrage qui sera fait tout conformement au présent projet voila le seul et l'unique moyen pour faire reussir notre entreprise.

Non signé

(Olivier de Vézain ?)

«Société entre les interessés en l'établissement des forges des Saint Maurice. », 16 Octobre 1736. A.P.C., Serie C 11 A, Canada, For ges de Saint Maurice, vol. 110, tome 1, pp. 241-251.

Nous soussignes François Etienne Cugnet premier Conseiller au Conseil Supérieur de Québec Directeur du Domaine d'occident en Canada, Thomas Jacques Taschereau Conseiller au dit Conseil supérieur de Québec, Trésorier de la marine au dit pays, Pierre François Olivier de Vezain maître de forges, Jacques Simonnet et aussi maîtrè de forges et Ignace Gamelin négociant à Montréal reconnoissons et confessons avoir de notre gré et volonté fäit traitté de société et Compagnie pour l'exploitation des mines de fer dont le privilège avoit été accordé au feu Sieur Francheville par brevet de $\mathrm{Sa} \mathrm{Ma}$ jesté du 25 mars 1730. et arrest du Conseil d'état du Roy du 4. Avril du dit an et qui a eté cedé a nous dits Cugnet Olivier de Vezain Simonnet et Gamelin par la veuve du dit feu Sieur Poulin 
de Francheville et par le sieur Pierre Poulin pour les parts et portions qui leur en appartenoient suivant l'acte de cession passé devant Pinguet et son confrère Notaires à Quebec le quinze du présent mois d'octobre pour continuer par notre société l'établissement des forges et l'exploitation des dites minës de fer aux termes du privilège nou' veau que nous avons suplié Sa Majesté de nous accorder par l'acté de soumission signé de nous dits Cugnet, Olivier de Vezain et Gamelin en datte du vingt trois Octobre mil sept cent trente cinq, de laquelle société contractée entre nous par les présentes il sera dressé acte en forme aux charges clauses et conditions des articles suivans dont nous sommes. convenus pour le fondement et clauses essentielles et principales de notre dite Société.

Le present traitté de Société est fait entre nous pour tous le tems du privilège qui nous sera accordé et ne finira quaprès l'expiration du dit privilège, a la fin duquel il nous sera loisible de continuer la dite société ou de la dissoudre et pourra chacun de nous garder pour soi le dit établissement de forges et exploitation des dites mines en remboursant a celuy ou ceux de nous qui sortiront de la dite société leur part et portion des effets, batimens, ustancils et matieres appartenant alors a la Compagnie suivant l'inventaire qui en aura eté arresté par la Compagnie.

\section{2}

La présente société sera composée de ving sols dont il appartiendra deux sols au dit Sieur Taschereau, et le surplus sera partagé entre nous dits Cugnet, Olivier de. Vezain, Simonnet et Gamelin en quatre parts et portions égales pour participer aux profits et pertes qu'il plaira a Dieu de donner a la dite société a raison des intérests cy dessus stipuler. jouir et disposer par chacun de nous nos heritiers, successeurs et aiant; cause et a la charge de faire chacun en droit soi les fonds qui seront régles a raison de son intérest en la présente société.

Pour marquer a Monsièr Hocquart intendant en cette colonie notre reconnoissance de la bonté qu'il a eue de contribuer de sa 
protection a l'etablissement des forges de fer et de nous' procurer les avantages que Sa Majesté a bien voulu nous accorder; nous sommes convenus et convenons par ces présentes d'offrir a mon dit Sieur Hocquart un intérest dans notre société d'un cinquieme dans les dix huit sols partagés entre nous dits Cugnet, Olivier de Vezain, Simonnet et Gamelin a prendre par portions egales sur nos interests, lequel intérest mon dit Sieur Hocquart sera prié d'accepter dans un an de ce jour aux memes clauses et conditions du présent traitte de société, et au cas qu'il ne l'accepte point nos dits intérest resteront entre nous suivant qu'ils sont stipules au précédent article, l'interest du Sieur Taschereau demeurant toujours fixé a deux sols.

Aucuns de nous ne pourra ceder son intérest total dans la pré sente société que du consentement et par délibération de toute la Compagnie qui aura la préférance a tous autres pour prendre l'in térest qu'aucun de nous voudra céder en remboursant au cédant son intérest dans la société sur le pied du dernier inventaire qui aura esté arresté.

Pourra néeanmoins chacun de nous ceder a qui bon luy semblera partie de son intérest, sans que les cessions puissent avoir voie déliberative en la dite société ny y faire aucune fonction en qualité d'associé.

\section{6}

Toutes les sommes qui nous seront avancées des deniers de Sa Majesté ainsi qu'elle a bien voulu nous l'accorder seront remboursées par la société solidairement et par chacun de nous suivant son intérest.

\section{7}

La direction et administration des forges sera tenue par le Sieur Olivier, lequel en conséquence sera chargé de la conduite tant de l'établissement et construction du fourneau, forges, affineries, chaufferies, fonderies, harnois et Bastimens.nécessaires pour l'exploitation des dites mines, ainsi qu'il a commencé de s'en charger depuis le 
mois de may dernier, que des retenues et chemin d'eau, fouilles de terres, coupes de bois fabrication et transport de charbons, amas et transport de mines, cassines et autres matières de la suite des travaux des ouvriers employés à l'exploitation des dites forges, et générallement de tout ce qui pourra concerner l'établissement et l'exploitation des dites mines et forges de fer, et cependant tout le tems que la présente société durera.

Le Sieur Simonet sera pareillement chargé de la direction et administration des dites forges de concert avec le dit sieur Olivier lorsqu'ils seront tous deux présens et en l'absence de l'un, l'autre en demeurera seul chargé, en sorte qu'ils ne pourront quittes tous deux en meme tems, et que l'un deux sera obligé de résider en l'absence de l'autre.

\section{9}

En consideration de quoy et de l'utilité dont les dits Sieurs Olivier et Simonnet seront a la société nous sommes expressement convenus qu'outre leurs intérest cy dessus stipuler au deuxieme article Ils auront : sçavois le Sieur Olivier trois mille livres et le dit Sieur Simonnet quinze cens livres d'appointemens par chaque année qui seront pris après toutes les autres dépenses prélevées sans que les dits appointemens puissent faire partie de frais de Regie de la présente société ny que les dits Sieurs Olivier et Simonnet puissent etre tenus de leur parts personnelle des dits appointemens a raison de leur intérest, mais seulement sera tenu le dit Sieur Olivier de sa part des appointemens du Sieur Simonnet Et le Sieur Simonnet de sa part de ceux du Sieur Olivier.

Tous les autres frais d'exploitation de Regie, transport et commerce, gages d'ouvriers et appointemens de commis frais de dépenses généralement quelconques concernant la dite société seront suportes par la compagnie et preleves par préferance a toutes choses avant de pouvoir procéder a aucun partage. 
Le Remboursement a faire a Sa Majesté des sommes qui nous auront eté avancées de ses deniers sera pareillement prélevé par preference avant de pouvoir faire entre nous aucun partage des profits que la dite société pourra produire.

\section{2}

Les appointemens des dits Sieurs Olivier et Simonnet courront meme pendant les voyages qu'ils pourront faire en france pour leurs affaires particulières, pourveu que les dits voyages ne soient : que pour un an, et au cas qu'ils restassent absent plus d'une année pour leurs affaires particulières, les dits appointemens cesseront jusqu’a leur retour.

\section{3}

Les dits Sieurs Cugnet et Gamelin se chargent de gérer toutes les affaires de la société scavoir lé dit Sieur Cugnet a Quebec et le dit Gamelin a, Montreal sans prétendre pour eux aucuns appointemens, jusqu'a ce que les affaires de la société deviennent assez considérables pour déterminer la Compagnie a leur accorder.

\section{4}

Les clauses ordinaires pour les délibérations plan de regie, tenue de registres, redditions de compte et confections d'inventaires et autres clauses générales de société seront reglées et stipulées par le traitté de société qui sera rédigé par les dits Sieurs Cugnet, Tachereau Olivier et Gamelin dans tout le mois de janvier prochain, au: quel le dit Sieur Simonnet souscrira a son retour de france, les articles cy dessus ayant eté reglées et convenus entre nous comme particuliers a notre société et faisans le fondement d'icelle.

Fait cinquième a Quebec le seize Octobre mil sept cent trente six.

Simonet. Cugnet. Olivier de Vezain, Ignace Gamelin, Tasche' reau.

«Traitté de Société des forges de Saint Maurice. », 1737. A.P.C., Série C 11 A, Canada, Forges de Saint Maurice, vol. 110, tome 1, pp. 252-321. 
Nous soussignés François Etienne Cugnet Premier Conseiller au Conseil Supérieur de Quebec Directeur du Domaine d'Occident en Canada demeurant a Quebec rue Saint Pierre, Thomas Jacques Taschereau conseiller au dit Conseil supérieur de Quebec Trésorier de la Marine au dit pays demeurant au dit Quebec rue du Parloir, Pierre François Olivier de Vezain maitre de forges demeurant ordinairement a Saint Maurice de présent en cette ville tant pour moy que pour le Sieur Jacques Simonnet ayssi maitre de forges de présent en france et Ignace Gamelin Negociant à Montréal demeurant ordinairement en cette ville, sommes convenus de ce qui suit, sçavoir, que Sa Majesté aiant par brevet du 25 mars 1730. accordé au feu Sieur François Poulin de Francheville la permission tant pour luy que pour les héritiers et aiant cause de faire l'ouverture des mines de fer qui se trouveront dans l'étendue de pays depuis et compris la seigneurie d'hyamachiche jusques et compris la seigneurie du cap de la Madeleine et de les faire fouillet et travailler a son profit a l'exclusion de tous autres et d'y faire construire les forges fourneaux et autres ouvrages qu'il conviendra pendant le tems de vingt années consécutives seulement à compter du jour de l'ouverture des dites mines qu'il seroit tenu de faire dans l'espace de deux années lors prochaines du jour de l'enregistrement du dit brevet au Conseil Supérieur de Quebec sans que les propriétaires des terres sur lesquelles les dites mines seroient ouvertes y puissent rien pretendre a la charge seulement de leur rembourser le prix des terres qui seroient cultivées suivant l'estimation qui en seroit faite par experts convenus entre lui et les dits propriétaires, ou qui seroient nommés d'office et sans qu'il fut tenu à aucun remboursement pour l'ouverture et exploitation des dites mines sur les terres qui n'auroient point été cultivées, lui permettant aussy Sa Majesté de faire les prises et retenues d'eau nécessaires a la dite exploitation dans les endroits et sur les terres qui se trouveroient les plus commodes a condition d'indemniser pareillement les propriétaires des terres sur lesquelles les dites prises et retenues d'eaux seroient faites s'Il y avoit lieu'a dé. dommagement et ce a dire d'experts convenus ou nommés d'office, sans que pour raison de la dite exploitation le d. Sieur Francheville fut tenu de paier a Sa Majesté ni a ses successurs rois, aucune finance ni indemnité pour quelque cause et sous quelque prétexte que ce soit. dont Sa Majesté lui a fait et remise même du dizième 
a elle appartenant et par arrest du Conseil d'Etat du 4 avril 1730. Sa Majesté aiant commis Monsieur l'Intendant de la nouvelle france, ou en son absence Monsieur le Commissaire de la Marine ordonnateur au dit pais pour connoître en première instance des proces différens, et contestations qui pourroient intervenir entre le dit Sieur Francheville pour raison du privilège exclusif a lui accordé pour l'ouverture des dites mines de fer et les propriétaires des terres sur lesquelles les dites mines seroient ouvertes ou sur lesquelles il seroit fait des prises et retenues d'eau pour servir a l'exploitation des dites mines, iceux juger sommairement et sans frais attribuant a cet effet toutte cour, jurisdiction connoissance pour raison de ce et icelle interdisant à toutes ses cours et autres juges sauf l'appel au Conseil de Sa Majesté avec permission a mon dit S. L'intendant, ou commissaire ordonnateur de subdéléguer sur les lieux telles personnes qu'ils aviseroient pour faire l'instruction des procès qui naitroient a l'occasion de l'ouverture des mines, les dits brevet $\mathcal{E}^{2}$ arrest du Conseil d'Etat enregistrés au Conseil Superieur de Québec; lequel privilège le dit Sieur Francheville auroit cédé a la société formée entre lui et le Sieur Poulin son frèr, François Etienne Cugnet, Louis Frédéric Bricaud de Valmur et Ignace Gamelin par traitté de société passé sous leurs signatures privées le 16 janvier 1733. reconnu par acte passé devant Pinguet Notaire a Québec le même jour, pour jouir par la compagnie du dit privilège aux termes des dits brevet et arrest du Conseil d'Etat, et en conséquence du dit traitté de société après avoir arresté le compte des dépenses faites jus qu'alors par le Sieur Francheville pour parvenir à l'exploitation des dites mines montant a $9244 \# 9^{3} 5^{\mathrm{d}}$ dont chacun des intéressés rem. boursa au dit Sieur Francheville sa part et portion suivant son intérest en la dite Société. le nommé La Breche fut envoié par la Compagnie a la nouvelle Angleterre avec les nommés La Palme forgeron et Bell'Isle charpentier pour examiner toutes les forges de fer établies au dit pays y prendre toutes les instructions nécessaires pour l'exploitation des forges de Saint Maurice, et en rapporter les mémoires et plans exacts, sur lesquels mémoires et plans rapportés par le dit La Brèche le Sieur Francheville fit construire a Saint Maurice une forge a la façon de celles de la nouvelle Angleterre une maison pour loger les ouvriers, et autres Bastimens nécessaires, et fit fondre a cette forge environ deux milliers de fer en barre; Sa Majesté aiant 
bien voulu è la meme année 1733 accorder au Sieur Francheville, une avance de dix mille livres pour lui faciliter les moiens d'exploiter les dites mines, cette somme lui a été remise pour en faire l'employ pour le bien et avantage de la société, ainsi qu'il avoit été convenu par le septième article du dit. Traitté de société, et-le $\mathrm{S}$. Francheville consentit obligation de la dite somme au profit de $\mathrm{Sa} \mathrm{Ma}$ jesté devanit Louet Notaire a Québec le 11 Octobre 1733. Le Sieur Francheville etant décédé au commencement du mois de Décembre 1733. La veuve Francheville consentit obligation en son nom au profit de Sa Majesté de la dite somme de dix mille livres devant Gaudron de Chevremont Notaire a Montréal le 19 du même mois. les Sieurs Cugnet et Gamelin se rendirent à Saint Maurice en Janvier 1734 avec la dite veuve Francheville pour convenir ensemble de ce qu'il y avoit a faire pour la suite de leur entreprise, et aiant reconnu qu'il étoit impossible de continuer l'exploitation de cette forge faute d'ouvriers experts et entendus Ils résolurent d'abandonner l'entreprise jusqu'a ce qu'ils eussent pû faire venir de france les ouvriers nécessaires, de laisser seülement deux gardiens à Saint Maurice pour veiller a la conservation des Bastimens, et des meubles apparteriant a la Compagnie, è de prier Monsieur Hocquart Intendant de rendre compte a Monseigneur le Comte de Maurepas de la situation de cet établissement le Sieur Gamelin arresta avec la veuve Francheville le 18 juillet 1734 le compte général extrait des comptes remdus par le feu Sieur Francheville et par sa veuve des dépenses faites aux forges de Saint Maurice jusqu'au dernier janvier de la même année montant a 21583 \#11 $1^{\mathrm{s}} 6^{\mathrm{d}}$. Monsieur Olivier de Vezain aiant été envoié par Monseigneur le Comte de Maurepas en l'année 1735. pour visiter les dites.mines de fer, et vérifier le succès qu'il y avoit lieu d'en espérer, le dit Sieur Olivier après avoir visité et vérifié les dites mines a dressé un projet des dépenses.a faire pour un établissement de forges capable de procurer les avantages que l'on peut attendre de l'exploitation des dites mines, et les intéressés aiant reconnu par ce projet qu'ils etoient hors d'état de continuer l'exploitation des dites mines a cause des sommes considérables qu'il étoit nécessaire' d'avancer pour l'établissement des forges et que les établissement faits par le Sieur Francheville pour la dite société ne pouvoient servir a ceux proposés par le Sieur Olivier ils auroient par acte passé devant pinguet Notaire à Québec le 23 octobre 1735 


\section{L'ACTUALITÉ ECONOMIQUE}

remis a Sa Majesté et mains de Monsieur Hocquart intendant le privilège accordé au Sieur Francheville par le brevest du 25 mars 1730 et arrest du Conseil d'état du 4 avril au dit an pour en disposer par Sa Majesté ainsi qu'elle le jugeroit a propos se désistant du dit privilège et renonçant a tous les droits qu'ils ont et peuvent avoir et prétendre en vertu du dit Brevet, et arrest du Conseil d'état, comm'aussi cédé et remis a Sa Majesté ou a ceux a qui elle voudroit accorder le privilège la seigneurie de Saint Maurice achetée par la Compagnie aux termes du traitté de société du 17 Janvier 1733. a constitution de trois cens livres de rente rachetable au principal de six mille livres, ensemble tous les Bastimens, la forge, les harnois de la dite forge, dalles et chemins d'eaux, maisons, meubles, outils, mine, charbon, ustancils, et tous les effets meubles et immeubles généralement quelconques étans pour lors au lieu de l'établissement fait sur le ruisseau de Saint Maurice par le dit feu Sieur Francheville au moien de laquelle cession et abandon, les dits intéressés supplioient Sa Majesté de vouloir les decharger des obligations consenties par le dit feu Sieur Francheville, et par la Demoiselle sa veuve les 11 Octobre et 19 Décembre 1733 de la dite somme de dix mille livres et de toutes autres dettes que le dit Sieur Francheville avoit pû contracter envers Sa Majesté pour raison des dites mines du ' paiement de laquelle somme de dix mille livres et autres dettes la dite veuve Francheville et ses dits associés demeureroient bien et valablement déchargés envers Sa Majesté sans pouvoir en être inquiétes ni recherchés a l'avenir et ordonner que les dits intéressés soient remboursés chacun suivant son intérest en la dite société du 16 Janvier 1733 de touttes les dépenses par eux faites pour l'établissement de forges de Saint Maurice, suivant le compte qui en seroit réglé et arreste avec eux par qui et ainsi qu'il appartiendroit, et qu'il seroit passé par ceux a qui seroit accordé le privilège des dites mines au profit du Sieur Pierre Poulin et autres copropriétaires de la seigneurie de Saint Maurice, et a la décharge des dits intéressés contract de constitution de trois cens livres de rente rachetable au principal de six mille livres, prix auquel les dits intéressés avoient acheté la dite Seigneurie par l'article 31 du traitté de société du 16 janvier 1733. moiennant quoi les dits intéressés promettoient renoncer au privilège accordé par les dits Brevet et arrest du Conseil des 25 mars et 4 avril 1730. et a tous les droits qu'ils pouvoient 
avoir et prétendre tant sur la dite Seigneurie de Saint Maurice que sur tous les établissemens faits au d. lieu pour l'exploitation des dites mines, maisons, meubles, ustancils, mine charbon et autres effets meubles et immeubles génerallement quelconques y étant, le meme jour de la Cession faite par les dits intéressés, les Sieurs $\mathrm{Cu}$ gnet, Olivier et Gamelin auroient remis a Monsieur Hocquart un acte sous leurs signatures privées par lequel en conséquence de la cession ils offrirent a Sa Majesté et se soumettoient de se charger de l'établissement des forges de Saint Maurice pour l'exploitation des mines de fer suivant le projet dressé par Monsieur Olivier et de continuer d'exploiter les dites mines s'il plaisoit a Sa Majesté en révoquant le privilege accordé au feu Sieur Francheville, par les dits brevet et arrest du Conseil d'état des 25 mars et 4 Avril 1730. leur accorder un nouveau privilège des dites mines aux mêmes termes clauses et conditions des dits Brevet, et arrest du conseil d'état pendant le tems et espace de vingt années consécutives a compter du jour que le fourneau auroit été chargé pour la première fois dont seroit dressé procès verbal par Monsieur le Lieutenant général de la jurisdiction des trois Rivières et leur prêter les fonds nécessaires suivant le dit projet, tant pour l'établissement et construction du fourneau de fonte, forges affinières, chaufferies, harnois et bastimens necessaires pour l'exploitation des dites mines que pour les dépenses annuelles de la dite exploitation, lesquels fonds jusqu'a concurrence de la somme de cent mille livres leur seroient remis au fur et a mesure qu'ils en auroient besoin sçavoir dans la premiere année pour la construction des fourneaux forges, harnois et Bastimens dans la seconde année pour la fabrication et transport de mine, castines, et autres matières, et dans la troisième pour les gages des ouvriers, au moien de quoy ils promettoient et s'obligeoient de rembourser a Sa Majesté tant la somme de dix mille livres pretée au feu Sieur Fran. cheville, suivant les obligations des 11 octobre et 19 Décembre 1733. et autres sommes qui pourroient avoir été avancées des de' niers de Sa Majesté, pour raison des dits mines, que pour les som. mes qui seroient prétées de nouveau par Sa Majesté, et d'en faire le remboursement en fers provenant des dites forges, ou de telle autre manière qu'il plairoit a Sa Majesté, et au cas qu'elle acceptât le remboursement en fers promettoient de les fournir a Quebec a raison de dix livres par millier au dessous du prix qu'ils sont ache'- 
tés en france pour la Marine de tel échantillon qu'ils leur seroient demandés a l'exception néantmoins des fers de fonderie qui ne pourroient leur etre demandés qu'après qu'ils auront etably une fonderie a commencer le premier remboursement dans la premiere année que les forges auroient travaillé et produit du fer, et continuer d'année en année jusqu'a parfait remboursement, pour la sureté desquels remboursement ils promettoient consentir obligation au profit de Sa Majesté de la dite somme de dix mille livres prêtée au feu Sieur Francheville, et des autres sommes qu'ils recevroient au fur et a mesure qu'elles leur seroient remises paiables aux termes cy dessus sous l'hipotèque des bastimens et fonds des forges et des fers qui en proviendront, et généralement de tous leurs biens meubles et immeubles présens et avenir, et s'obligeoient pareillement de rembourser a la société du Sieur Francheville les dépenses par elle faites jusqu'alors a l'occasion des dites mines et de passer contract de constitution de trois cens livres de rente rachetable au principal de six mille livres pour l'acquisition de la Seigneurie de Saint Maurice aux termes du dit acte de cession faite par la Société du dit Sieur Francheville, lequels actes de cession et de soumission des dits Sieurs Cugnet Olivier et Gamelin envoiés par Monsieur Hocquart à Monseigneur le comte de Maurepas, Sa Majesté a bien voulu accorder aux dits Sieurs Cugnet Olivier et Gamelin, les avances par eux demandées pour l'établissement des forges et les ordres du Ministre aiant été reçus au commencement du mois de Juillet 1736. le dit Sieur Olivier a fait construire a Saint Maurice un fourneau de fonte, avec une forge, affinerie et chaufferie lesquels ouvrages ont été trouvés bien établis par le Sieur Simonnet Maitre de forges envoié par Monseigneur le Comte de.Maurepas pour travailler au dit établissement de concert avec le Sieur Olivier, et y prendre intérest si bon lui sembloit, ce que le dit Sieur Simonnet a accepté et fait continuer avec le dit Sieur Olivier les dits ouvrages sur le plan et suivant les établissemens commencés par le dit Sieur Olivier; en conséquence de l'acte de cession faite a Sa Majesté par les intéressés en la Société du Sieúr Francheville, de la soumission et offres des Sieurs Cugnet, Olivier et Gamelin et des ordres de Sa Majesté la demoiselle veuve Francheville et le Sieur Pierre Poulin intérresses au traitté de société du 16 janvier 1739. sçavoir la demoiselle Francheville pour cinq dixième, et le: dit. Sieur Pierre Poulin pour 
un dixième ont par acte passé devant Pinguet et Boisseau Notaires a Québec le 23 Octobre 1736. Remis, cede, quitté et transporté aus Sieurs Cugnet, Olivier, Simonnet et Gamelin les Parts et portions. appartenantes aux d'elle Veuve Francheville et S. Pierre Poulin dans le privilège accordé a feu sieur Francheville par les brevet et arrest des 25 mars et 4 avril 1730. en conséquence du dit traitté de société du 16 janvier 1733. pour en jouir et disposer par les dits cessionnaires leurs hoirs et aians cause se désistant les dits Sieur et demoiselle cedant de leurs parts et portion dans lesdit privilège au profit des dits cessionnaires et renonçant a tous les droits généralement qu'ils avoient et prétendre en vertu des dits brevet et arrest du Conseil d'état, et traitté de société des 25 Mars et 4 Avril 1730 et 16 janvier 1733. et ont pareillement cédé aux dits Cugnet, Olivier, Simonnet et Gamelin la seigneurie de Saint Maurice, ensemble tous les Bastimens, la forges, les harnois de la dite forge, dalles et chemins d'eaux, maisons, meubles et immeubles généralement quelconques. etans au lieu de l'établissement fait par le Sieur Franche' ville sur le Ruisseau de Saint Maurice pour prise de.laquelle cession les dits Sieurs Cugnet, Olivier Simonnet et Gamelin ont promis solidairement paier et rembourser a Sa Majesté a l'acquit de la demoiselle Veuve Francheville, la somme de dix mille livres avancés des deniers de Sa Majesté au feu Sieur Francheville suivant les obliga. tions des 118 'bre et 19 Decembre 1733. et de touttes autres dettes que le Sieur Francheville a pû contracter envers Sa Majesté pour. raison des dite mines, promettant de faire donner dans le jour par Monsieur Taschereau Trésorier de la Marine en ce pais a la demoiselle veuve Francheville quittance de la somme de dix mille livres en sorte que la dite demoiselle Francheville et le Sieur Pierre Poulin n'en puissent etre inquiétés ny recherces a l'avenir, les dits Sieurs Cugnet, Olivier, Simonnet et Gamelin demeurant obligés solidairement de les indemniser, et en outre de paier et rembourser aux $d$. demoiselle veuve Francheville et Sieur Pierre Poulin sçavoir a la demoiselle Francheville la somme de six mille huit cent cinquante sept livres deux sols sept deniers a laquelle montent les avances faites tant par le dit feu Sieur Francheville que par elle an la dite société compris la somme de quatre cens cinquante livres pour la rente de la seigneurie de Saint Maurice pendant trois ans a laquelle somme de six mille huit cent cinquante sept livres deux sols sept deniers 
a été reglé par provision le remboursement a faire à la demoiselle veuve Francheville, sauf sans préjudice du compte final a régler entr'elle et les dits Sieurs Cugnet et Gamelin pour les sommes qu'ils ont paiées respectivement pour la dite société dont le compte incessament arresté, et au dit Pierre Poulin la somme de neuf cens vingt quatre livres huit sols onze deniers a laquelle a eté reglé définitivement son contingent a lui rembourser et en outre les sits Sieurs Cugnet, Olivier Simonnet et Gamelin ont promis de passer incessament et a la volonté du dit Sieur Pierre Poulin, et copropriétaires de la Seigneurie de Saint Maurice contrat de constitution de la somme de trois cens livres de rente rachetable au principal de six mille livres pour l'acquisition de la seigneurie, le même jour 15 octor bre 1736. Les Scurs Pierre Poulin et demoiselle Louise le Boulan, ger son épouse de lui duement autorisée, et Messire Michel Poulin Prestre Chanoine de l'eglise Catedrale de Québec Missionnaire a Beauport ont par acte passé devant Pinguet et Boisseau Notaires a Quebec vendu avec promesse solidaire de garantir de tous troubles, dettes, hipotèques, dons souaires, evictions, aliénations, et autres empêchement généralement quelconques aux dits Sieurs Cugnet, Olivier, Simonnet et Gamelin présens et acceptant acquéreurs pour eux leurs hoirs et aians cause le fief nommé Saint Maurice contenant une lieue de front sur la Rivière des Trois Rivières a prendre du costé du sud ouest aux confins de la seigneurie appartenante aux héritiers Lafond en descendant la dite rivière sur deux lieues de profondeur avec droit de pesche sur la dite rivière vis a vis la dite lieue de front, circonstances et dépendances, sans par les d. Sieurs vendeurs en rien réserver ni retenir. Quand même la seigneurie se trouveroit de plus grande etendue, la dite vente faite a la charge par les acquéreurs des droits et redevances dont le fief se trouve envers le domaine de Sa Majesté et moiennant le prix et somme de six mille livres de prix principal, laquelle somme de six mille livres du consentement des vendeurs est demeurée en mains des acquéreurs qui l'ont retenu a constitution de trois cent livres de rente annuelle et perpetuelle rachetable a toujours. Que les dits acquéreurs ont promis et se sont obligés solidairement paier aux dits vendeurs ou aians cause jusqu'au parfait rachat d'icelle qui ne pourra être fait qu'en un seul paiement et par le dite somme principale de six mille livres avec les arrérages qui seront dûs lors du 
rachat ; la dite rente échéante au premier jour de janvier de chaque année dont il est déclaré être dû au premier janvier de la présente année la somme de sept cent cinquante livres pour arrérages de la dite rente, attendu la possession que les acquéreurs ont ê̂ cy devant du dit fief etans convenus avec la demoiselle veuve Francheville de lui paier la somme de quatre cent cinquante livres pour arrérages de la même rente a elle due suivant l'acte passé entr'elle et les dits sieurs acquéreurs le même jour, et pour sureté de la dite rente en principal et arrérages les dits acquéreurs ont solidairement obligé et hipotéqué tous leurs biens généralement quelconques presens et futurs, et specialement le dit fief sans que l'hipotèque générale et spéciale dérogent l'une a l'autre, et le 16 du dit mois d'octobre les dits Sieurs Cugnet Olivier Simonnet et Gamelin ayant asso cié le sieur Taschereau. Ils sont avec le dit Sieur Taschereau con: venus par acte passé sous leurs signatures privées du $16 \mathrm{du}$ dit mois d'Octobre de passer entre eux dans le mois de janvier de la présente année un traitté de société sur le fondement des clauses particulières contenues au dit acte en treizes articles pour continuer par la dite société des Sieurs Cugnet, Taschereau, Olivier, Simonnet et Gamelin l'établissement des forges de Saint Maurice et l'exploitar tion des dites mines de fer aux termes du nouveau privilège demandé a Sa Majesté par l'acte de soumission des dits Sieurs Cugnet et Olivier et Gamelin en datte du 23 octobre 1735. auquel traité de société le dit Sieur Simonnet souscrira a son retour de france pour l'execution desquelles conventions arrestées entre nous le 10 octobre dernier, et des autres actes mentionnés cy devant Nous reconnoissons et confessons avoir fait de notre gré et volonté E.faisons ensemble le présent traitté de société et Compagnie pour l'exploi: tation des dites mines de fer aux termes du privilège que nous espérons obtenir de Sa Majesté, et ce pour tous le tems du dit privilège a commencer de ce jour jusqu'a l'expiration du dit privilège aux clauses et conditions des articles suivans, Prians Dieu en etre le conducteur et y donner ses saintes bénédictions.

La présente société sera composée de vingt sold dont il appartiendra deux sold au dit Sieur Taschereau et le surplus sera par. 
tagé entre nous dits Cugnet, Olivier; Simonnet et Gamelin en qua. tre parts et portions égales pour participer aux profits et pertes qu'il plaira a Dieu de donner a la dite société a raison des intérests cy dessus stipulés jouir et disposer par chacun de nous de nos parts et portions cy dessus pour nous nos heritiers successeurs et aians cause, et a la charge de faire chacun en droit soy les fonds qui seront réglés a raison de son intérest en la présente société.

\section{2}

Etant convenus par le troisième des articles arrestés entre nous le 16 octobre dernier d'offrir a Monsieur Hocquart intendant en cette Colonie un intérest en la présente société dans les dix huit sols partagés entre nous d. Cugnet Olivier Simonnet et Gamelin au cas que Monsieur Hocquart áccepte le dit intérest, ce qu'il a été prié de faire dans un an du jour des dits articles signés, nous convenons que le dit intérest sera pris par portions égales sur ceux appar' tenant a nous d. Cugnet, Olivier Simonnet et Gamelin, aux mêmes clauses et conditions du présent Traitté de société et que le partage sera fait après l'acceptation de Monsieur Hocquart par délibération de' la Compagnie et au cas qu'il ne l'accepte point, nos intérests resteront entre nous suivant qu'ils sont stipules au précédent àrticle l'intérest du dit Sieur Taschereau demeurant toujours fixé a deux sols.

\section{3}

Aucun de nous ne pourra céder son intérest total dans. la présente société que du consentement et par délibération de toutte la Compagnie qui aura la préference a tous autres pour prendre l'intérests qu'aucun de nous voudra céder en remboursant au cédant son intérests dans la société sur le pied du dernier inventaire qui aura esté arresté.

\section{4}

Pourra néantmoins chacun de nous céder a qui bon luy sem. blera partie de son intérest sans que les cessionnaires puissent parô̂. tre en la présente société y avoir voix délibérative, ni y faire aucune fonction en qualité d'associé. 
Aucun de nous ne pourra entreprendre ny former en cette colo nie aucuns nouveaux établissement de forges que pour l'utilité et le profit de la présente société, et seront tenus ceux de nous qui pourront projetter quelques nouveaux établissements de forges en ce pais de communiquer leurs projets à la Compagnie pour en suivre l'éxecution si elle est jugée avantageuse du consentement et par délibération de toutte la Compagnie par preference a toute autre nouvelle société Sauf a y intéresser les nouveaux associés que l'auteur ou auteurs des projets pourroient proposer, a peine contre celuy ou ceux de nous qui auroient formé de nouveaux projets, et de nouvelles sociétés pour l'execution d'iceux sans en avoir donné connoissance a la Compagnie de perte de leurs intérests en la présente société qui seront et demeureront acquis de plein droit a la Compagnie en vertu du présent article sans qu'elle soit tenue de leur en faire aucun remboursement, nonobstant laquelle perte de leurs inté rests ils resteront toujours obligés solidairement a touttes les dettes passives que la Compagnie aura pû contracter jusqu'alors comme si leurs intérests leur étoient conservés, et seront en outre tenus de tous les dépens dommages et intérests de la Compagnie sans prée judice des mesures qu'elle jugera apropos de prendre pour obtenir la suppression des nouveaux etablisemens qui pourroient luy etre préjudiciables.

\section{6}

Les fonds par nous faits en la présente société consistent seulement aux dépenses faites par la société du Sieur Francheville contenues en l'état arresté par les intéresses en la dite société le 16 jan' vier 1733 mentionné au huitièmé article du dit traitté de société du dit joưr 16 Janvier: et en l'état signé du Sieur Francheville du 15 juillet de la meme année lesquelles dépenses la Compagnie s'est chargée de rembourser a la dite société du Sieur Francheville aux termes de la soumission des Sieurs Cugnet Olivier et Gamelin du 23 octobre 1735 dont les parts et portions des dits demoiselle veuve Francheville et Sieur Pierre Poulin leur ont effectivement ete remboursées aux termes de la cession par eux faite le 15 octobre dernier suivant leurs quittances des 26 du dit mois d'octobre, et 28 
Décembre aussi dernier, et aux avances que Sa Majesté a bien voulu faire tant au feu Sieur Francheville de la somme de dix mille livres dont nous nous sommes charges d'acquitter la demoiselle veuve Francheville qu'a la présente société, desquelles avances nous avons consenti obligation au profit de Sa Majesté devant Pinguet et $\mathrm{Ba}$ rolet Notaires a Quebec le 18 octobre dernier pour la somme de $52963 \# 16^{3} 5^{d}$

\section{7}

Et attendu que nous n'avons point fait d'autres fonds en la présente société, et que Sa Majesté a bien voulu nous avancer les fonds nécessaires pour perfectionner l'établissement des forges et l'exploitation d'icelles nous convenons qu'il sera ouvert a chacun de nous suivant nos intérests cy dessus stipules un compte courant en débit et crédit au livire de raison qui sera tenu par le Sieur Cugnet sur lequel compte courant chacun de nous sera débité suivant son intérest de la part et portion dont il est et sera débiteur a la société pour les sommes qui ont déjà été et seront par la suite avancées des deniers de Sa Majesté pour etre crédité chacun aussy suivant son intérest des sommes qui seront remboursées a Sa Majesté sur le produit des forges au fur et a mesure du remboursement qui en sera fait.

Les Sieurs Cugnet et Gamelin n'aiant point été remboursés par la présente société des sommes par eux fournies a la société du Sieur Francheville pour leur contigent en icelle, ils seront chacun à leur égard crédités au dit compte courant des sommes qu'ils seront trouvés avoir paiées de leurs deniers a la dite société suivant le compte final qui doit etre réglé et arresté incessament entre la veuve Francheville et les dits Sieurs Cugnet et Gamelin aux termes du dit acte du 15 octobre dernier.

\section{9}

Monsieur De Valmur sera porté débiteur a la société au dit livre de caisse de la somme de 1047 \# $^{\text {s }}$ qu'il doit a la Compagnie pour solde de son dixième d'intérest en la société du 16 Janvier 1733 suivant son billet du 19 octobre dernier attendu que la société a rem- 
boursé pour lui la demoiselle veuve Francheville et le Sieur Pierre Poulin.

Sa Majesté aiant bien voulu, ainsi qu'il est expliqué cy devant, nous accorder les avances nécessaires pour l'établissement des forges les sommes qui nous ont été et seront avancées des deniers de Sa Majesté seront remboursées par la société solidairement et par chacun de nous suivant son intérest.

\section{1}

Les fonds nécessaires pour les dépenses annuelles de l'exploitation des forges seront pris sur le produit des dites forges, et pour cet effet il sera réglé par délibération de la Compagnie et arresté chaque année dans le mois de Septembre un état des frais d'exploitation transport et commerce, gages d'ouvriers et appointemens de commis, frais et dépenses généralement quelconques concernant la dite société qui auront été faits pendant l'année jusqu'au premier du dit mois de septembre pour constater la somme qui sera prélevée sur le produit des forges pendant la dite année pour servir aux dépenses de l'année suivante.

\section{2}

Jusqu'a ce que toutes les avances qui nous ont été faites des deniers de Sa Majesté soient remboursées en entier ce qui restera du produit des forges excedant l'etat des dépenses annuelles de l'exploitation mentionnée au précédent article sera emploié au remboursement a faire a Sa Majesté et ne sera fait aucun partage des profits que la Société pourra produire qu'après le dit remboursement fait en entier.

\section{3}

Après le dit remboursement fait en entier a Sa Majesté de touttes les sommes qui auront été avancées a la Société, il sera paié aux sieurs Pierre Poulin et Michel Poulin copropriétaires de la Seigneurie de Saint Maurice la somme de six mille livres pour le rachat de la rente a eux constituée par le contract de vente du 15 octobre 
dernier pour prix d'acquisition de la dite seigneurie après lesquels paiement faits, l'ecédant du produit au dela de l'état qui aura été arresté pour les dépenses de l'année suivante aux termes de l'article onze sera réputé profit net et pourra par délibération de la Compagnie etre partagé entre nous suivant nos intérests cy dessus régles.

\section{4.}

Au cas que dans le cours de la présente société la Compagnie soit obligée d'emprunter, ce qui ne pourra etre fait que par délibération expresse de toute la Compagnie, chacun de nous sera obligé au paiement des dits emprunts en principal et intérest si intérests y a solidairement et l'un de nous seul pour tous, bien entendu que la présente clause dé solidité ne vaudra que pour les emprunts qui auront été régles par délibération expresse de toute la compagnie et non pour ceux qui pourroient etre faits par un ou plusieurs de nous sans délibération auxquels emprunts faits sans délibération seront seulement obligés ceux de nous qui les auront faits.

\section{5}

Ne sont comprises au précédent article les avances que nous recevrons de Sa Majesté pour l'établissement des forges jusqu'a ce qu'elles soient entièrement établies et en train de travailler aux' quelles avances nous demeurerons obliges solidairement et l'un de nous seul pour tous aux termes dé l'article dix du présent Traitté de société encoor que les dites avances n'eussent pas été reçues en vertu de délibération de la Compagnie mais sera seulement entendu le dit précédent article des emprunts qui pourroient etre faits dans le cours de la société après l'établissement entièrement finy.

\section{6}

S'il est jugé a propos de faire des fonds en marchandises pour achat de vivres, charbon, mines, castines et autres matières gages d'ouvriers, journaliers, et entrepreneurs d'ouvrage, la quantité et qualité des marchandises sera réglé par délibération de la compagnie sur les mémoires qui lui en seront envoiés par les Sieurs. Olivier et Simonnet, ou l'un d'eux en l'absence de l'autre et seront les dites 
marchandises demandées en france ou achetées à Quebec pour' le compte de la Compagnie aux meilleures conditions que faire se pourra, et après l'arrivée ou achat des dites marchandises il sera arresté une facture des prix auxquels la Compagnie aura reglé quie les dites marchandises seront vendues ou données en paiement aux ouvriers journaliers, fournisseurs et entrepreneurs d'ouvrages-auxquelles factures ceux qui vendront ou donneront en paiement les dites marchandises se conformeront.

Le Sieur Cugnet aiant demandé a Bordeaux au Sieur Jung trente barriques de vin et quarante quarts d'eau de vie pour le compte de la Société, les dits vins et eaux de vie viennent pour compte et ris' ques de la Société.

\section{8}

La raison de la présente société sera tenue sous le nom de compagnie des forges de Saint Maurice et seront tous actes, marches, lettres et billets de change, ordres sur icelles, et autres billets et promesses paiables a ordre, endorsement des lettres ou billets' de change, promesses et billets a ordre, ou autres pour en recevoir le paiement, quittances et autres actes concernañt la présente société timbrés de ces mots, Compagnie des forges de Saint Maurice, et seront tenus ceux qui les signeront de les signer de leur nom et compagnie. faute de quoy les dites lettres de change, et billets de change, billets et promesses a ordre, endorsements, quittances, marchés et autres actes seront réputés pour le compte particulier de celuy qui les aura signés sans que la Compagnie puisse en etre responsable.

\section{9}

La direction et administration des forges sera tenue par le Sieur Olivier lequel en conséquence demeure chargé de la conduite tant de l'établissement et construction du fourneau, forges, affineries harnois et Bastimens nécessaires pour l'exploitation des dites mines, ainsi qu'il a commencé de s'en charger depuis le mois de May dernier. que des retenues et chemins d'eaux fouilles de terre, coupes de bois, fabrication et transport de charbon, amas et transport de 


\section{L'ACTUALITE ECONOMIQUE}

mines, castine et autres matières, marchés, et execution d'iceux, inspection des travaux, des ouvriers et engagés a l'exploitation des dites forges, nourriture et paiement des dits ouvriers et engagés et généralement de tout ce qui pourra concerner l'établissement et exploitation des dites forges et cependant tout le tems que la présente société durera.

\section{0}

Le dit Sieur Simonnet sera pareillement chargé de la Direction et administration des dites forges de concert avec le dit Sieur Olivier lorsqu'ils seront tous les deux présens, et en l'absence de l'un, l'autre en demeurera seul chargé, en sorte qu'ils ne pourront quitter tous deux en même tems et que l'un d'eux sera obligé de résider en l'absence de l'autre.

\section{1}

En considération de quoy et de l'utilité dont les dits Sieurs Oli' vier et Simonnet seront a la société nous sommes expressément convenu qu'outre leurs intérests cy dessus stipulés au premier article du présent traitté de société, ils auront sç̧avoir le dit Sieur Olivier trois milles livres a commencer du premier Avril 1735. et le dit Sieur Simonnet quinze cents livres a commencer du premier aoust 1736 . d'appointemens par chaque année qui seront emploies dans les comptes après touttes les dépenses prélevées sans que les dits appointements puissent faire partie des frais de régie de la presente société ni que les dits Sieurs Olivier et Simonnet puissent etre tenus de leur part personnelle des dits appointemens a raison de leur intérest.

\section{2}

Tous les autres frais d'exploitation, de régis, achats, transport, et commerce, gages d'ouvriers et appointemens de commis, frais et dépenses généralement quelconques concernant la dite société seront supportés par la Compagnie et prélevées par préférence a touttes choses avant de pouvoir procéder a aucun partage.

Les appointemens des dits Sieurs Olivier et Simonnet courront même pendant les voiages qu'ils pourront faire en france pour leurs 
affaires particulières pourvû que les dits voiages ne soient que pour un an, et au cas qu'ils restassent absens plus d'une année pour leurs affaires particulières les dits appointemens cesseront jusqu'a leur retour.

\section{4}

La caisse de la Société a Saint Maurice sera tenue par le dit Sieur Olivier et en son absence par le dit Sieur Simonnet sans que pour raison de ce ils puissent prétendre d'autres appointemens que ceux stipulés par l'article vingt un cy dessus, ny qu'ils puissent de' mander a la Compagnie aucune choses pour les abus qui pourroient y arriver si ce n'est pour diminution d'espèces, au cas de suppression de la monnoie de cartes ou pour cas fortuis d'incendie, ou de vol par effraction auxquels cas la perte sera supportée par la Com. pagnie dont sera arresté une délibération, comm'aussi en cas d'aug. mentation d'espèces le profit en appartiendra a la Compagnie.

\section{5}

Le dit Sieur Olivier et en son absence le dit Sieur Simonnet en la dite qualité de Directeurs et Caissiers signeront les marchés qu'il sera nécessaire de faire pour tirer la mine, coupes de bois, fournitures de charbon, bâtimens nécessaires aux dites forges, engagemens d'ouvriers, mandats billets sur la caisse de Québec, et généralement tous les actes qu'il conviendra faire a Saint Maurice et dans toute l'étendu et dans toute l'étendue du Gouvernement des Trois Ri, vières pour le service de la société lesquels marchés et engagemens, et autres actes ils timbreront et signeront conformément a l'article dix huit des présent Traitté de Société et envoieront au Sieur $\mathrm{Cu}$. gnet a Quebec l'extrait des marches et engagemens qu'ils auront faits a l'effet d'etre enregistres sur son livre de raison.

\section{6}

Le dit Sieur Olivier et en son absence le dit Sieur Simonnet fe' ront en la dite qualité les paiemens tant pour fournitures de vivres et fourages, charbon, mines, castines et autres matières que pour gages d'ouvriers, journaliers et autres dépenses:courantes suivant les 
marchés qui auront été faits par eux ou par les Sieurs Cugnet et Gamelin.

\section{7}

Les marchandises dont il aura été fait fonds aux termes de l'ar' ticle seize du présent traitté de société seront remises au dit Sieur Olivier, et en son absence au Sieur Simonnet pour etre emploiées au paiement des gages d'ouvriers et journaliers, fournitures et autres dépenses.

\section{8}

Le dit Sieur Olivier ou en son absence le dit Sieur Simonnet tiendra un registre journal en bon ordre sur lequel il écrira jour par jour par ordre de datte sans aucun blanc ni interligne la recette des fonds qui lui seront remis par la Caisse de Quebec tant en argent qu'en marchandises desquelles marchandises sera porté sur le pied de la facture d'achat et la dépense qui sera par lui faite des fonds tant en argent qu'en marchandises au prix qu'il aura été convenu de les vendre conformement a l'article seize du présent traitté de société en distinguant ce qu'il aura paié en marchandises, a qui les paiemens auront été faits et pour quelles causes, c'est a dire si c'est pour fournitures de charbon, mines castines et autres matières journées d'ouvriers, gages salaires et autres dépenses.

Les vivres qui seront remis de Quebec au dit Sieur Olivier seront portés sur le dit journal en gros en recette et dépense. sçavoir pour la recette la quantité qu'il en aura reçûe pour la dépense la quantité qui en aura été consommée.

\section{0}

Le dit Sieur Olivier tiendra un autre journal sur lequel il écrira jour par jour par ordre de datte et sans aucun blanc la quantité de mine, charbon, castines et autres matières qui auront eté fournies aux dites forges et la quantité de fer en fonte, et en barre que les dites forges produiront. 
Et un autre journal sur lequel il écrira jour par jour la quantité et qualité des fers qu'il envoiera au Sieur Cugnet a Quebec et au Sieur Gamelin a Montreal pour y etre vendus ou qu'il aura vendu aux trois rivières.

Le Sieur Gamelin sera chargé a Montreal de faire les achats de vivres et marchandises qui lui seront demandés par le Sieur Olivier ou en son absence par le Sieur Simonnet, et par le Sieur Cugnet, et de faire la vente des fers qui lui seront envoiés par le Sieur Olivier ou le Sieur Simonnet pour être vendus a Montreal et tiendra un registre journal sur lequel il ecrira jour par jour par ordre de datte et sans aucun blanc les recettes et dépenses qu'il fera pour compte de la Société tant en argent et marchandises qu'en fers qui luy auront été envoiés sans que pour raison de ce le dit Sieur Gamelin puisse pretendre aucuns appointemens, si ce n'est que par la suite la Compagnie juge devoir luy en accorder par délibération, ni que le dit Sieur Gamelin puisse demander aucune chose a la Compagnie pour les abus qu'il pourroit faire dans sa gestion et maniement ou pertes qui pourroient y arriver, si ce n'est pour diminution d'es pèces en cas que la monnoye de cartes fut supprimée en ce pais, ou pour cas fortruit d'incendie ou de vol par effraction auxquels cas la perte sera supportée par la Compagnie dont sera arresté une délibération de même qu'en cas d'augmentation d'espèce le profit appartiendra a la Compagnie.

La Direction et caisse de la Société sera tenue a Québec par le dit Sieur Cugnet sans que pour raison de ce ils puissent prétendre aucuns appointemens, si ce n'est que par la suite lorsque le Commerce de la Société sera bien etably la Compagnie juge devoir lui . en accorder par délibération ni que le dit Sieur Cugnet puisse demander a la Compagnie aucune chose pour les abus qu'il y pourront faire ou pertes qui pourroient y arriver. si ce n'est pour diminution d'espèces, en cas que la monnoie de cartes fut supprimée en ce pais ou pour cas fortruit d'incendie ou de vol par effraction, aux' 


\section{L'ACTUALITE ECONOMIQUE}

quels cas la perte sera supportée par la Compagnie dont sera arresté une délibération de même cas d'augmentation d'espèces, le profit appartiendra a la Compagnie.

Le dit Sieur Cugnet en la dite qualité de Directeur et Caissier de la Société a Québec signera les marchés, engagemens, mémoires pour commission des marchandises en france, lettres et billets de change et promesses paiables a ordre, endossemens de lettres ou billets de change, promesses et Billets a ordre ou autres pour en recevoir le paiement, quittances, et généralement tous autres actes touchant la présente société qui seront faits a Québec tous lesquels billets et actes le dit Sieur Cugnet sera tenu de timbrer de ces mots, Compagnie des forges de Saint Maurice, et signer Cugnet et Compagnie, faute de quoy les dits billets seront réputés pour son compte particulier sans que la Compagnie puisse en etre responsable.

\section{5}

Le Sieur Cugnet fera tous les paiemens qui seront a faire. a Québec pour achats de marchandises; vivres, gages et salaires d'ouvriers et autres depenses suivant les marchés qui en auront eté faits par le Sieur Olivier, et par le Sieur Simonnet en son absence ou par luy ou sur les mandats et ordres des Sieurs Olivier et Simonnet.

\section{6}

Les procès et contestations contre tous redevables a la Compagnie qui pourront intervenir pour raison du privilège a elle accordé seront poursuivis par le dit Sieur Cugnet devant Monsieur l'Intendant ou Commissaire ordonnateur sans pouvoir par lui faire aucun accommodement ny transaction, ny accorder aucune remise ni main levée en cas de saisie que du conșentement et par délibération expresse de la Compagnie.

Le dit Sieur Cugnet tiendra un registre de la Caisse en bon or dre, sur lequel seront écrites toutes les Recettes et dépenses qui 
seront par lui faites tant en argent marchandises ou fers qui lui seront envoiés par le Sieur Olivier, et en son absence par le Sieur Simonnet qu'en remise de lettres et billets de change, et autres paiables a ordre le tout jour par jour, par ordre de datte, sans aucun blanc rature ni interligne, et seront les dites recettes et depenses arrestees en chaque article a la fin.

\section{8}

Et un livre de raison sur lequel seront portées en debit et crédit touttes les parties écrites, tant sur son journal de caisse et sur les journaux des Sieurs Olivier ou Simonnet et Gamelin mentionnés aux articles vingt huit, trente, trente un, et trente deux du présent traitté de société que pour chacun de nous associés et pour ceux qui seront débiteurs ou créditeurs a la Compagnie pour ventes, achats, emprunts, gages, salaires ou autres raisons que ce puisse être.

\section{9}

Et pour l'exécution du présent article le Sieur Olivier et en son absence le Sieur Simonnet et le dit Gamelin envoieront tous les trois mois au dit Sr Cugnet un extrait ou copie des dits registres journaux mentionnes aux articles vingt huit, trente, trente un et trente deux du présent traitté de société pour etres les parties acrites sur les dits journaux porté au dit livre de raison.

\section{0}

- Le dit Sieur Cugnet tiendra un registre des délibérations de la Compagnie pour y écrire jour par jour par ordre de datte et sans aucun blanc les délibérations de la Compagnie.

\section{1}

Les registres mentionnés au présent traitté de société seront cot: tés et paraphés par le Sieur Taschereau par premier et dernier feuil. let, et ceux tenus par le Sieur Cugnet seront arrestés année par année au premier Septembre par les dits Srs. Taschereau et Cugnet, et ceux tenus par les dits Sieurs Simonnet et Olivier, et par le dit Sieur Gamelin seront aussi arrestés année par année par eux au premier septembre. 


\section{2}

Il ne sera fait aucun emprunt, achat considérable, établissement voiages pour compte de la société, ni pris aucune résolution importante pour les affaires de la Société que par délibération de la Compagnie.

\section{3}

Tout ce qui n'aura point été prévu par le présent Traitté de Société sera réglé par délibération de la Compagnie aux termes du présent traitté de société.

\section{4}

Et attendu que nous sommes résidens scavoir les dits Sieurs Cugnet et Taschereau a Québec, les d. Sieurs Olivier et Simonnet a Saint Maurice, et le Sieur Gamelin a Montréal, il est convenu que nous nous écrirons respectivement les affaires sur lesquelles il sera nécessaire de délibérer en marquant précisément et décisivement nos avis par nos lettres, et que les délibérations seront redigées à la pluralité des voix et portées sur le registre des délibérations a Québec par les Sieurs Cugnet et Taschereau avec la copie entière des lettres des sieurs Olivier Simonnet et Gamelin comtenans leurs avis sur les dits délibérations, lesquelles seront signées par les dits Srs Cugnet et Taschereau, au moien de quoy elles vaudront comme si elles étoient Signées par toutte la compagnie.

\section{5}

Il sera dressée arresté tous les ans a la fin du mois d'aoust par les Sieurs Olivier et Simonnet un inventaire de tous les effets de la société étans a Saint Maurice, contenant le détail des bastimens, meubles, bestiaux, outils, ustancils, mine, charbon, castines, Bois de corde, fers marchandises, et autres effets géneralement quelconques appartenans a la Compagnie au dit lieu de Saint Maurice et aux trois Rivières, lequel inventaire sera envoié au Sieur Cugnet dans les premiers jours de septembre.

\section{6}

Il sera pareillement dressé et arresté par le Sieur Gamelin et envoié au Sieur Cugnet dans le meme tems un inventaire de tous les effets appartenans a la Compagnie a Montreal. 
Il sera aussi dressé et arresté par les Sieurs Cugnet et Taschereau dans le même tems pareil inventaire de tous les effets appartenans a la Compagnie a Quebec.

\section{8}

Les inventaires mentionnés aux articles quarante cinq, quarante six et quarante sept précedent serviront pour la reddition des comptes a rendre par les dits Sieurs Olivier, Simonnet, Gamelin et $\mathrm{Cu}$ gnet sans qu'il soit nécessaire d'autres comptes que les dits inven' taires lesquels seront a cet effet vérifiés et arrestés par la Compagnie sur les livres desquels ils auront été extraits et pièces justificatives d'iceux tous les ans dans le mois de février pourquoy les Sieurs Cugnet et Gamelin se rendront tous les ans dans le dit mois de février à Saint Maurice pour y arrester, et signer les dits inventaires respectifs avec les Sieurs Olivier et Simonnet.

\section{9}

Sur lesquels inventaires, et sur le livre de raison mentionné en larticle trente huit du présent traitté de société il sera arresté tous les ans dans le mois de septembre un inventaire général de tous les effets tant actifs que passifs de la Société contenant le détail des bastimens, meubles, bestiaux, outils, ustancils, mine, charbon, cas tines, bois de corde, fers, marchandises et autres effets généralement quelconques appartenans a la Compagnie tant à Saint Maurice et aux trois Rivières, qu'a Montreal et a Quebec, et des dettes actives et passives de la Compagnie Par noms et surnoms des débiteurs et créditeurs qui seront extraits du dit livre de raison et sera fait au tant de copies du dit inventaire que nous sommes d'aaosies dont sera remis une copie a chacun de nous.

\section{0}

Après l'expiration du privilège accordé a la Compagnie et du présent traitté de société il sera loisible a la Compagnie de continuer la présente société, et en cas de dissolution de la dite société, chacun de nous pourra demander pour luy la continuation du dit privilège 
et exploitation des mines en remboursant a chacun des intéresses sa part et portion des effets Bastimens, ustancils et matières générale ment tous meubles et immeubles appartenans alors a la Compagnie suivant l'inventaire qui en aura été arresté par la Compagnie si la société continuera ou sera resolue un an avant l'expiration du privilège.

Tous les différens et contestations qui pourra survenir entre nous dans le cours de la présente société, ou lors de la dissolution d'icelle, et pour raison de la dite societe circonstances et dépendances seront terminés a l'amiable sur le fondement du présent traitté par deux personnes choisies et nommées par la Compagnie lesquelles ne le pouvant accorder leur donnons pouvoir dès a présent comme des lors de prendre et nommer un tiers au jugement desquels nous nous remettons dès a présent comme dès lors promettant de les executer comm'arrest de cour souveraine a peine contre les contrevenans de six mille livres applicables moitié a l'hopital des trois rivières, et l'autre moitié aux acquiesçans sans pouvoir appeller des dits jugemens qu'après avoir préalablement paié et remis a la Caisse de la Compagnie la dite somme de six mille livres, et ne pourront la dite clause ni touttes celles contenues au présent traitté de société portant peines a etre réputées commiratoires attendu que nous nous y sommes soumis et soumettons de gré et volonté et quelles font partie de la présente société qui n'auroit contracttée sans icelles.

Le présent traitté de société devant subsister pendant tout le tems du dit privilège qui sera accordé a la compagnie le dit présent traitté de société est contracté entre nous pour tout le dit tems tant pour nous que pour nos héritiers, successeurs et aiant cause, lesquels deront soumis et obligés de même que nous a toutes et chacunes des conditions y énoncées.

\section{3}

Arrivant le décès de l'un de nous, son intérest passera comme dit est a l'article précédent a sa veuve, enfans, héritiers, successeurs et ayans cause. lesquels pour ne point multiplier le nombre de vois 
dans les délibérations de la Compagnie seront tenus de nommer un d'entr'eux ou une personne pour eux a l'effet de représenter la succession et tenir lieu et place de leur auteur pour la voix délibéra: tive seulement, non pour remplir les employs et fonctions que leurs auteurs auroient pû exercer de leur vivant, lesquels emplois et fonc. tions demeureront a la disposition de la compagnie.

\section{4}

Au cas que l'un de nous se trouve obligé de quitter la Colonie et passer en france pour toujours, et qu'il ne puisse ou ne veuille conserver son intérest dans la présente société son intérest lui sera remboursé par la Compagnie aux termes de l'article trois du pré sent traitté de société pour par la Compagnie disposer du dit intérest ainsi qu'elle le jugera a propos.

\section{5}

Le présent traitté de société devant servir de base et de fondement a notre société et a touttes les délibérations qu'il conviendra prendre pour la régie d'icelle suivant que nous en somme convenus, et convenons par le présent article le dit traitté sera transcrit tout au long sur chaque registre des délibérations au commencement des dits registres afin que nous puissions y avoir un recours toujours présent dans toutes nos délibérations.

56

Nous sommes convenus de donner aux pauvres tous les ans une somme qui sera réglée par délibération de la Compagnie en égard a la situation de la société laquelle somme séra donnée aux pauvres malades de l'hopital des trois Rivières.

\section{7}

Il sera entretenu un missionnaire a Saint Maurice pour y faire le service divin dans la chapelle qui sera bâtie a cet effet, et administrer les sacremens aux ouvriers, journaliers, et a tous ceux qui résideront au dit lieu, et sera le missionnaire logé, nourri et chauffé aux depens de la compagnie et en outre lui sera paié par la Compagnie deux cens livre par chacun an. 
58 et dernier

Et pour l'entière execution dés présentes en tout leur contenu nous promettons les uns aux autres amitié et fidélité, et de maintenir le présent traitté sans aucun innovation en touttes les clauses et conditions y mentionnées et aux peines y portées à quoy nous avons obligé et obligeons mutuellement les uns envers les autres tant pour nous que pour nos veuves, enfans, héritiers successeurs et aians cause tous nos bien meubles et immeubles généralement quelconques présent et avenir Nous soumettans et nos dites veuves, enfans, héritiers, successeurs et aians cause a tous jugemens d'arbitres aux termes de l'article cinquante du présent traitté, et aux clauses et conditions enoncées au dit présent traitté sous le dit obligation cy dessus aux peines y portées et a tous dépens dommages et intérests, élisans nos domiciles irrévocables en nos demeures ordinaires auxquels nous voulons que tous actes en conséquences soient faits valablement pour l'exécution du présent Traitté de société. qui sera reconnu par devant Notaires, et copie d'iceluy déposée pour minutte en l'etude du notaire devant qui il sera reconnu.

Fait cinquième et signé par nous Cugnet Olivier et Gamelin a Saint Maurice le onze février mil sept cent trente sept, et par mois Taschereau a Québec le vingt du dit mois au dit an.

Cugnet 'Taschereau

Ignace Gamelin

Olivier de Vezain

« Etat des fonds nécessaires a l'exploitation des forges de Saint Mau* rice. », A.P.C., Série C 11 A, Canada, Forges de Saint Maurice, vol. 110 , tome 1 , pp. 345-351.

\section{Sçavoir}

Guages des forgerons

2 Marteleurs a 800 \#. l'un par an $1600 \#$

9 Chauffeurs à 600\# par an 5400 .

6 Gouyards a 300\# par année 1800.

Un charron a 600 \# par an 600 .

9809\# 
LA BANQUEROUTE DE FRANÇOIS ÉTIENNE CUGNET, 1742

200 \# pour vins aux forgerons 200.

250 \# de suif a graisser

les mouvemens

125.

84\# pour les fetes de Saint Eloy

aux forgerons

84.

Consommation de charbons pour les forges.

1800 Bannes de charbon à 20 bari-

ques l'une du prix de 9 \# la Banne

cy

$16000 \#(\text { sic })^{5}$

300 \# pour la charois des gueuses

16500.

aux forges et aigilles

$300 \#$

Guages et consommation pour le fourneau.

Un fondeur à 900 \# par an 900 \#

Un second fondeur servant de guarde 900 .

4 chargeurs pendant 8 mois a $30 \# 960$.

un casseur de castine
a 25 \# par mois
200.
un maistre mouleur
600.

2 aydes mouleurs a 30 \# par

4498

mois pour 3 mois chacun

180.

un chartier pour le fourneau

avec son harnois à $76 \#$

par mois pour 8.

608.

$100 \#$ de suif pour graiffer

les mouvemens

50.

100 \# pour vins de fourneaux 100.

Nombre de bannes de charbon pour faire un million 500 milliers de fonte.

\section{Sçavoir}

Suite des depenses du fourneau.

1600 Bannes de charbon

a 9 \# la Banne

$14400 \#$

5. Le renvoi à da prochaine page est de $16200 \#$. 
2400 pipes de mines

a $50^{s}$ l'une

6000

8 toizes de pierre a chaux pour cas tine a 40 \# de la toise rendue au fourneau

6 toizes de pierres a chaux pour

ouvrages et parois au fourneau

800 Bariques de chaux a 3 \#

l'une pour id.

100 Bariques de sable a $3 \#$

100 \# pour autres petits faux frais imprévus cy

1800 \# pour le transport des fers aux trois Rivières a Quebec et a Montreal

300 \# pour le transport id. des poèles plaques de poèles marmites et autres cy

Frais de Régie

Pour appointemens du Directeur, un commis, un ecrivain un missionnaire un chirurgien 2 chevaux entretenus avec leurs équipages, pour l'usage du commis l'enmagasinage et reception des fers aux trois Rivières et frais d'embarquement cy $9000 \#$ 6500 \# tant pour le chauffage des forgerons fondeurs et autres et $y$ compris la maison, que pour réparation et entretien des forges et du fourneau relevage des soufflets et achapt de ballance avec leurs entre tien cy 


\section{Sçavoir}

200 milliers de fer vendu dans la Colonie a $20 \#$ le mille cy $40.000 \#$

400 milliers de fer pour les arceneaux de Sa Majesté a 15\# le mille cy

60000.

100 milliers en marchandises, poeles, plaques de poe' les, marmites, chaudières et autres a 20 \# le mille 20000 .

$120000 \#$

\section{Récapitulation}

des depenses annuelles cy

Profit celle de

69722.

50.278

Pour pouvoir Monseigneur retirer ce profit certain il faut doubler les fonds de dépenses annuelles la premiere année, qui monteroit selon ce projet a 139444\#. de laquelle somme il doit toujours rester à ces forges, un fond de fer de 60000 \#. en souffrance, c'est-adire en appret de fonte, mines castines charbons et autres matières, ce qui faciliteroit les dépenses annuelles, a estre diminuée selon le projet et a pouvoir indubitablement encore les réduire par la suitte pourvu que le Directeur ait plein pouvoir d'establir sa regie, comme il convient, ce qui feroit d'abord une différence de 26278 \# d'epargne par chacune année sur la dépense annuelle de 96000 a laquelle pernitieux commerce de marchandises avoit fait monter celle de 1740. pendant l'absence du dit Olivier en france, cause aussi par le mauvais ordre qui avoit eté tenu a la forge et par les gages et nourriture considérables d'un nombre de commis inutiles qui aug' mentoient les frais de régie de plus de 3000 \#. que ceux spécifiés par le Memoire auquel le dit Olivier ose se flatter Monseigneur, de donner son execution, sous le bon plaisir et la protection de Votre Grandeur en voulant bien luy accorder de remener en Canada un fondeur capable d'y faire les fontes necessaires en marchandises, 4 goujards pour le service des forges et achettez en france le Tuyaires de cuivre rongé pour l'usage de la dite forge dont la dépense sera prise sur celle mentionnée en cet estat de dépenses. 


\section{LACTUALTE ECONOMIQUE}

Esperant én outre Monseigneur que Votre Grandeur voudra bien accorder au dit Olivier une gratification proportionnée aux dépenses qu'il a eté obligé de faire pour venir rendre compte de cet etablissement a Votre Grandeur.

Non signé

(Olivier de Vézain) $^{\circ}$

\section{Cameron NISH, professeur à l'Université Sir George Williams et}

Chargé de Recherche, Centre de Recherche d'Histoire Economique H.E.C.

6. Bien que le texte ne soit pas signé il est évident qu'il est de la plume de Vézain; l'année i 740 paraît être la date'à assigner à cette convention. 